\title{
Impact of gas heating in inductively coupled plasmas
}

\author{
D. B. Hash, a) D. Bose, ${ }^{\text {b) }}$ M. V. V. S. Rao, ${ }^{\text {b) }}$ B. A. Cruden, ${ }^{\text {b) }}$ M. Meyyappan, \\ and S. P. Sharma \\ NASA Ames Research Center, Moffett Field, California 94035
}

(Received 29 March 2001; accepted for publication 14 June 2001)

\begin{abstract}
Recently it has been recognized that the neutral gas in inductively coupled plasma reactors heats up significantly during processing. The resulting gas density variations across the reactor affect reaction rates, radical densities, plasma characteristics, and uniformity within the reactor. A self-consistent model that couples the plasma generation and transport to the gas flow and heating has been developed and used to study $\mathrm{CF}_{4}$ discharges. A Langmuir probe has been used to measure radial profiles of electron density and temperature. The model predictions agree well with the experimental results. As a result of these comparisons along with the poorer performance of the model without the gas-plasma coupling, the importance of gas heating in plasma processing has been verified. (1) 2001 American Institute of Physics. [DOI: 10.1063/1.1390503]
\end{abstract}

\section{INTRODUCTION}

Inductively coupled plasma (ICP) sources have recently become popular in semiconductor processing. They provide a high plasma density $\left(10^{10}-10^{12} \mathrm{~cm}^{-3}\right.$ ) and allow independent control of the plasma density and ion energy at the wafer surface. Plasma etching in an ICP reactor is a complex process characterized by strong interactions among plasma chemistry, surface chemistry, power coupling, plasma physics, and gas flow. Plasma diagnostics as well as reactor modeling are important in developing an understanding of reactive discharges employed in plasma processing. Such an understanding is valuable in equipment and process design, and process control.

Because of the low pressures at which ICPs and other plasma sources operate, simulation tools employed to acquire such insights may use a kinetic, fluid, or hybrid approach. The first self-consistent two-dimensional nonequilibrium simulations of a if glow discharge were published by Wu employing both a fully fluid model ${ }^{1}$ and a hybrid model ${ }^{2}$ with a Monte Carlo technique for electrons and a fluid representation for ions. Other particle electron-fluid ion simulations soon followed. The work of Ventzek $e t a l^{3}$ laid the groundwork for the widely used Hybrid Plasma Equipment Model code. ${ }^{4-10}$ Similarly, contributions were made to hybrid research by Fiala et al. ${ }^{11}$ During the same time period, Graves et $a l^{12-14}$ introduced a particle ion-fluid electron hybrid approach for simulating an electron cyclotron resonance plasma. A large number of research teams however have focused on the development of a fully fluid approach. ${ }^{1,15-33}$

In the early 1990s when two-dimensional simulations of radio frequency plasmas began to appear, most of the aforementioned research, whether hybrid or fluid, neglected the coupling between the neutral gas and the plasma which recent experiments $\mathrm{s}^{34}$ have shown to be important. There were a few exceptions. Ventzek et al. ${ }^{5}$ solved a decoupled hydrody-

\footnotetext{
"Author to whom correspondence should be addressed; electronic mail: hash (a) dm 3.arc.nasa.gov

b) With Eloret Corporation, Sunnyvale, CA 94087.
}

namics model to provide the gas velocity flow field. Mass, momentum, and energy conservation equations were employed but the energy equation did not include heating from inelastic collisions. Moreover, the only information used from the neutral gas simulation was the velocity field. They concluded that the effects of the gas flow on the global plasma parameters were secondary for low pressure systems. The Modular Plasma Reactor Simulator code of Lymberopoulos and Economou ${ }^{22}$ recognized the importance of gas-plasma coupling but only included mass conservation for the neutrals, neglecting momentum and energy conservation. The convective term $\nabla \cdot \rho \mathbf{u}$ in the mass conservation was approximated by a relaxation term $\Delta \rho / \tau_{\text {res }}$. Finally, Fiala $e t a l .{ }^{27}$ included mass and momentum equations for the neutral species but did not solve the neutral energy conservation.

All of this research neglected what is now seen as a significant effect in plasma processing, that of neutral gas heating. Gas temperatures on the order of $1000 \mathrm{~K}$ have been observed in an argon ICP at $240 \mathrm{~W}^{35} 1400 \mathrm{~K}$ in a $\mathrm{Cl}_{2} / \mathrm{BCl}_{3} / \mathrm{N}_{2}$ plasma at $1000 \mathrm{~W},{ }^{36}$ and $2100 \mathrm{~K}$ in a point-ofuse plasma abatement tool at $1200 \mathrm{~W} .{ }^{37}$ This effect has only recently been addressed in the modeling community by Bukowski et al. ${ }^{38} \mathrm{Nam}$ et al., ${ }^{39}$ and the CFD-PLASMA code under the direction of Kolobov at CFDRC in Huntsville, and Kiehlbauch and Graves. ${ }^{37}$ Bukowski et al. allow for gas heating through ion-neutral elastic and inelastic collisions, Franck-Condon heating, and ion-ion recombination. Nam et al. do include neutral gas conservation equations for mass, momentum, and energy. However, they make the assumption that the energy transfer from the plasma to the neutral gas through collisions is $5 \%$ of the deposited power in an ad hoc manner. Kiehlbauch and Graves present the most thorough representation to date of the true physics by modeling Franck-Condon heating, electron impact vibrational excitation of neutrals, gas phase exothermic reactions, ion-neutral gas heating through inelastic and elastic collisions, and ionion recombination. They show that in fact the power transferred to the neutrals in their $\mathrm{CF}_{4}$ work is more on the order 
of $25 \%-30 \%$ of the rf power. But this of course is dependent on the gas itself and the reaction mechanisms involved. Regardless, it is now evident that detailed modeling of the actual energy transfer mechanism is necessary for accurate simulations.

The purpose of the present research is to further the understanding of the importance of gas heating in plasma processing and also further validate the Semiconductor Equipment Modeling Software (SEMS) code under development at NASA Ames Research Center. $\mathrm{CF}_{4}$ mixtures have been extensively investigated in the Gaseous Electronics Conference (GEC) reference cell but none have been published in the ICP mode. The following sections describe the computational model and the experimental measurements of an $\mathrm{ICP}-\mathrm{CF}_{4}$ plasma in a GEC reference cell. Comparisons of the SEMS predictions to Langmuir probe measurements of electron temperature and density will then be used as the metric for code validation and for the the discussion of the importance of gas heating. Further experimental results and analysis are presented in two companion papers. The first ${ }^{40}$ characterizes the electron energy distribution function, and the second ${ }^{4} \mathrm{i}$ uses Fourier transform infrared spectroscopy to characterize the degree of $\mathrm{CF}_{4}$ dissociation which is therein compared to results from this work.

\section{COMPUTATIONAL MODELING}

SEMS consists of a comprehensive fluid model for simulating high-density plasma reactors. The model couples the plasma and neutral gas transport and heat transfer in a selfconsistent manner. No assumptions regarding the energy transferred from the plasma to the gas are made. Instead, similar to Kiehlbauch and Graves, ${ }^{37}$ the energy exchange mechanisms between the plasma and the gas are modeled. Details regarding the model have been presented previously ${ }^{42-44}$ but will be briefly addressed here. The model solves a coupled set of equations including mass conservation for each species, a total momentum equation, and thermal energy conservation equations for the neutrals and electrons.

\section{A. Species continuity}

$$
\frac{\partial \rho_{s}}{\partial t}+\nabla \cdot \rho_{s} \mathbf{u}=-\nabla \cdot \mathbf{J}_{s}+\sum_{r} R_{\mathrm{sr}}
$$

Here, the subscript $s$ denotes an individual species and $\rho_{s}$ its density. $\mathbf{u}$ is the mass averaged velocity of the mixture, and $\mathbf{J}$ is the diffusion mass flux, $\rho_{s} \mathbf{V}_{s}$, with $\mathbf{V}_{s}$ representing the species diffusion velocity. The diffusion fluxes are determined from the self-consistent effective binary diffusion formulation of Ramshaw and Chang. ${ }^{45-47}$ For the ions, this requires knowledge of the mobilities $\mu$ to determine the diffusivities $\mathcal{D}$ through the Einstein relation, $\mu=|e| / k T_{i} \mathcal{D}$, where $e$ is the elementary charge. The mobilities are determined from the Langevin formula ${ }^{48}$

$$
\mu_{i, n} n_{n}=\frac{3.73 \times 10^{22}}{\sqrt{\alpha_{n} m_{r}}}\left(\mathrm{~m}^{2} / \mathrm{Vs}\right),
$$

where the subscript $i$ represents a single ion species, the number density $n_{n}$ is for a single neutral species in $\mathrm{m}^{-3}$, the polarizability $\alpha_{n}$ is also for a single neutral species with units of $\AA^{3}$, and the reduced mass $m_{r}$ is measured in $\mathrm{g} / \mathrm{mole}$. The ion mobility is then determined from summing over all ionneutral interactions with Blanc's law

$$
\frac{1}{\mu_{i}}=\sum \frac{X_{n}}{\mu_{i, n}},
$$

where $X_{n}$ is the mole fraction of the neutral species $n$.

The subscript $r$ in the source term of the continuity equation denotes an individual reaction with the term $R_{\mathrm{sr}}$ representing the production of species $s$ from reaction $r$ defined as follows:

$$
\begin{aligned}
& R_{\mathrm{sr}}=M_{s}\left(\nu_{\mathrm{sr}}^{\prime \prime}-\nu_{\mathrm{sr}}^{\prime}\right) k_{r} \prod_{k}\left(\frac{\rho_{k}}{M_{k}}\right)^{\nu_{k r}^{\prime}}, \\
& k_{r}=A T^{\alpha} \exp \left(-E_{\mathrm{a}} / T\right) .
\end{aligned}
$$

$M$ is the molecular weight, and $k_{r}$ is the reaction rate constant in an Arrhenius form where $E_{a}$ is the activation energy and $T$ is the effective temperature for the reaction, i.e., ion, electron, or neutral temperature depending on the reaction. All reactions used in the simulation are fit to this form. When available, reaction cross sections are integrated over an assumed Maxwellian distribution to provide the reaction rates. These reaction rates are then curve fit to the Arrhenius form. The terms $\nu^{\prime}$ and $\nu^{\prime \prime}$ in the above equation are the stoichiometric coefficients for the reaction

$$
\sum_{s} \nu_{\mathrm{sr}}^{\prime} \chi_{s} \rightarrow \sum_{s} \nu_{\mathrm{sr}}^{\prime \prime} \chi_{s}
$$

where $X$ is the chemical symbol.

\section{B. Total momentum}

$$
\frac{\partial \rho \mathbf{u}}{\partial t}+\nabla \cdot \rho \mathbf{u u}=-\nabla p+\nabla \cdot \tau
$$

Here, $p$ is the total pressure, and $\tau$ is the shear stress.

$$
\begin{aligned}
& \text { C. Neutral thermal energy } \\
& \begin{aligned}
\frac{\partial \rho_{n} e_{n}}{\partial t}+\nabla \cdot \rho_{n} e_{n} \mathbf{u}= & -\nabla \cdot \sum_{s, \text { neutrals }}\left(\mathbf{q}_{s}+h_{s} \mathbf{J}_{s}\right)-p_{n} \nabla \cdot \mathbf{u} \\
& +\sum_{s, \text { neutrals }} \frac{\mathbf{J}_{s}}{\rho_{s}} \cdot \nabla p_{s}+Q_{\text {elastic }}+Q_{\text {ce }} \\
& +\sum_{r} \sum_{s, \text { neutrals }} R_{\mathrm{sr}} \Delta H_{r}^{\circ} .
\end{aligned}
\end{aligned}
$$

Here the subscript $n$ denotes a property of all the neutrals as a combined set, $e_{n}$ is the neutral thermal energy, $e_{n}$ $=\Sigma_{s \neq i, e} \rho_{s} C_{u, s} T, \mathbf{q}$ is the heat flux, and $h$ is the specific enthalpy. The terms $\nabla \cdot \rho_{n} e_{n} \mathbf{u}, \nabla \cdot \mathbf{q}$, and $\nabla \cdot h \mathbf{J}$ are the transport of energy due to convection, thermal conduction, and diffusion, respectively. The next two terms in the equation are due to pressure volume work. The remaining terms represent sources to the neutral thermal energy equation 
whereby the heating of the gas is modeled. The $Q_{\text {elastic }}$ term models the energy transfer due to elastic collisions between electrons and neutrals. The $Q_{\mathrm{ce}}$ term models the energy transfer due to charge exchange collisions between ions and neutrals. The $R_{\mathrm{sr}} \Delta H_{r}^{\circ}$ term models the chemical energy released or absorbed in a reaction where $\Delta H_{r}^{\circ}$ is the heat of reaction. This term then includes gas heating from ion-ion recombination, dissociative recombination, gas phase exothermic reactions, and associative detachment.

Viscous dissipation, neutral vibrational excitation, and the Franck-Condon effect are not considered. Kiehlbauch and Graves ${ }^{37}$ show viscous dissipation to be negligible. SEMS has the capability of modeling vibrational excitation but this was shown only to represent $10 \%$ of the heating of the neutral $\operatorname{gas}^{37}$ and is therefore also ignored. The Franck-Condon effect is a result of the quantized energy states allowable to a molecule. When an electron impacts a molecule because of the Franck-Condon rule there are only certain states possible for the molecule to achieve. If dissociation occurs as a result of the electron impact, this can result in extra energy being deposited into the molecule fragments resulting from the difference in the energy required for dissociation and the allowed excited state. Information concerning the additional energy funneled to the fragments is largely unavailable and thus has not been included in the present work.

\section{Electron thermal energy}

$$
\begin{aligned}
\frac{\partial \rho_{e} e_{e}}{\partial t}+\nabla \cdot \rho_{e} e_{e} \mathbf{u}= & -\nabla \cdot\left(\mathbf{q}_{e}+h_{e} \mathbf{J}_{e}\right)-p_{e} \nabla \cdot \mathbf{u}+\frac{\mathbf{J}_{e}}{\rho_{e}} \cdot \nabla p_{e} \\
& -\sum_{r \neq e} Q_{\text {elastic,s }}+\sum_{r} R_{e r} \Delta H_{r}^{\circ} \\
& +\frac{1}{2} j_{\theta} E_{\theta} .
\end{aligned}
$$

Here, the term $\frac{1}{2} j_{\theta} E_{\theta}$ is the power deposited into the plasma from the coil assuming fully collisional heating, $j_{\theta}$ is the azimuthal plasma current, and $E_{\theta}$ is the azimuthal electric field.

Maxwell's equations are solved separately to compute $E_{\theta}$ and $j_{\theta}$ where $j_{\theta}=\sigma E_{\theta}$ using Ohm's law with $\sigma$ being the plasma conductivity. The Maxwell's equations are then coupled at regular intervals to the conservation equations. For these results, a Poisson solver is not used, and the space charge induced electric fields are given by the Boltzmann relation assuming that the plasma is quasineutral. The most recent version of SEMS ${ }^{49}$ includes Poisson's equation and separate ion momentum equations to consider the important inertial effects at low pressure, but these capabilities are not utilized in the present work.

\section{CF $_{4}$ MODELING}

Fluorocarbon plasmas such as $\mathrm{CF}_{4}, \mathrm{CHF}_{3}$, and $\mathrm{C}_{4} \mathrm{~F}_{8}$ are used in oxide etching. Given the significance of this process to the microelectronics industry, there has been an emphasis on basic research to understand the characteristics of fluorocarbon plasmas. One of the main impediments to modeling has been either the lack of measurements of reaction cross sections or uncertainty in those that have been measured. For $\mathrm{CF}_{4}$, this is especially relevant for the reactions involving negative ions. ${ }^{50}$ The reaction set used in this work is summarized in Table I, consisting of electron impact, neutralneutral, and neutral-ion exchange reactions. The rates are given in Arrhenius form and are determined from integration over a Maxwellian distribution of the reaction cross sections when available. Construction of the reaction set in Table I assumes a negligible concentration of negative ions in a $\mathrm{CF}_{4}$ plasma and avoids the uncertainty in the electronegative reaction mechanisms. Experimental confirmation for the large presence or absence of negative ions in $\mathrm{CF}_{4}$ plasmas is scarce. In our work, a mass spectrometer with a positively biased extraction orifice was mounted on the side of the chamber to detect negative ions. While this approach disturbs the plasma and may not be representative of the true negative ion density, the negative ion flux was 2 orders of magnitude lower than the positive ion flux, suggesting the negative ion density is fairly small. For completeness, Table II lists additional reactions which need to be considered if $F^{-}$ is found in significant amounts; these reactions include dissociative attachment, polar dissociation, associative detachment, and ion-ion recombination. The effect of the inclusion of this reaction set is discussed in the results.

All the simulations are for a power input of $300 \mathrm{~W}$ with an estimated power absorption of $80 \%$ based on currentvoltage $(I-V)$ probe and reflected power measurements. The $\mathrm{CF}_{4}$ flow rate is $20 \mathrm{sccm}$, and the wall temperatures are fixed at $400 \mathrm{~K}$. The ion temperature is specified at $0.2 \mathrm{eV}$ for use in calculating the ion diffusivities. Three different pressures are considered: 10,30 , and $50 \mathrm{mTorr}$. The fluorocarbon gas is modeled with 13 species: $\mathrm{CF}_{4}, \mathrm{CF}_{3}, \mathrm{CF}_{2}, \mathrm{CF}, \mathrm{F}, \mathrm{C}, \mathrm{F}_{2}, \mathrm{CF}_{3}^{+}$, $\mathrm{CF}_{2}^{+}, \mathrm{CF}^{+}, \mathrm{F}^{+}, \mathrm{F}^{-}$, and $e$. All ions are neutralized upon impact with the wall, and atomic fluorine is allowed to recombine on the walls with a probability of $0.02 .^{51}$ The transport properties for the neutrals are determined using the Lennard-Jones parameters shown in Table III and the techniques in Gupta et al. ${ }^{52}$ The ion transport properties are determined using the polarizabilities given in the same table. and the electron transport properties are given from integrating the electron momentum transfer cross section presented in Christophorou et al. ${ }^{53}$

\section{EXPERIMENT}

Our experimental setup consists of a GEC reference cell modified for inductive coupling shown in Figs. 1-5. A detailed description of the apparatus, the Langmuir probe (LP) construction, and measurement procedures has been recently presented $^{54}$ and hence, only a brief account is provided here. The GEC cell is powered by a flat five-turn and 3.5 in. diam. inductive coil made of a $0.125 \mathrm{in}$. diam. copper tube. The inductive coil is mounted at the top of the chamber and is concentric with the center of the chamber to maintain discharge symmetry. The coil is insulated from the plasma by a quartz window of 0.375 in. thickness and 5 in. diameter. The water-cooled lower electrode (4 in. diameter) is made of 304 
TABLE I. $\mathrm{CF}_{4}$ reaction mechanism. Species: $\mathrm{CF}_{4}, \mathrm{CF}_{3}, \mathrm{CF}_{2}, \mathrm{CF}, \mathrm{C}, \mathrm{F}, \mathrm{F}_{2}, \mathrm{CF}_{3}^{+}, \mathrm{CF}_{2}^{+}, \mathrm{CF}^{+}, \mathrm{F}^{+}, \rho$

\begin{tabular}{|c|c|c|c|}
\hline Reaction & Rate coefficient $\left(\mathrm{cm}^{3} \mathrm{~s}^{-1}\right)$ & $\Delta H_{,}^{\circ}(\mathrm{eV})^{\mathbf{a}}$ & Reference \\
\hline$e+\mathrm{CF}_{3} \rightarrow \mathrm{CF}_{3}^{+}+2 e$ & $1.213 \times 10^{-9} T_{e}^{0.9076} \exp \left(-9.2727 / T_{e}\right)$ & 9.09 & Christophorou et al. ${ }^{53}$ \\
\hline$e+\mathrm{CF}_{2} \rightarrow \mathrm{CF}_{2}^{+}+2 e$ & $4.071 \times 10^{-9} T_{e}^{0.0029} \exp \left(-9.4004 / T_{e}\right)$ & 11.5 & Christophorou ef at. ${ }^{53}$ \\
\hline$e+\mathrm{CF} \rightarrow \mathrm{CF}^{+}+2 e$ & $3.621 \times 10^{-9} T_{e}^{0.8665} \exp \left(-11.198 / T_{e}\right)$ & 9.17 & Christophorou et al. ${ }^{53}$ \\
\hline$e+\mathrm{F} \rightarrow \mathrm{F}^{+}+2 e$ & $4.702 \times 10^{-9} T_{e}^{0.4344} \exp \left(-17.586 / T_{e}\right)$ & 17.5 & Meyyappan and Govindaul ${ }^{50}$ \\
\hline$e+\mathrm{CF}_{4} \rightarrow \mathrm{CF}_{3}^{+}+\mathrm{F}+2 e$ & $4.153 \times 10^{-9} T_{e}^{1.3554} \exp \left(-15.599 / T_{e}\right)$ & 14.8 & Christophorou et al. ${ }^{53}$ \\
\hline$e+\mathrm{CF}_{4}+\mathrm{CF}_{2}^{+}+2 \mathrm{~F}+2 e$ & $2.104 \times 10^{-9} T_{e}^{0.8103} \exp \left(-23.164 / T_{e}\right)$ & 20.8 & Christophorou et al. ${ }^{53}$ \\
\hline$e+\mathrm{CF}_{4} \rightarrow \mathrm{CF}^{+}+3 \mathrm{~F}+2 e$ & $2.729 \times 10^{-9} T_{e}^{0,7747} \exp \left(-33.101 / T_{e}\right)$ & 23.9 & Christophorou et al. ${ }^{53}$ \\
\hline$e+\mathrm{CF}_{3} \rightarrow \mathrm{CF}_{2}^{+}+\mathrm{F}+2 e$ & $9.001 \times 10^{-9} T_{e}^{0.4111} \exp \left(-17.463 / T_{e}\right)$ & 15.2 & Christophorou et al. ${ }^{53}$ \\
\hline$e+\mathrm{CF}_{3} \rightarrow \mathrm{CF}^{+}+2 \mathrm{~F}+2 e$ & $5.045 \times 10^{-9} T_{\varepsilon}^{0.5737} \exp \left(-21.174 / T_{\epsilon}\right)$ & 18.2 & Christophorou et al. ${ }^{53}$ \\
\hline$e+\mathrm{CF}_{2} \rightarrow \mathrm{CF}^{+}+\mathrm{F}+2 e$ & $4.737 \times 10^{-9} T_{e}^{0.6696} \exp \left(-14.310 / T_{e}\right)$ & 14.6 & Christophorou et al. ${ }^{53}$ \\
\hline$e+\mathrm{CF}_{4} \rightarrow \mathrm{CF}_{3}+\mathrm{F}+e$ & $3.257 \times 10^{-9} T_{e}^{0.6906} \exp \left(-14.370 / T_{e}\right)$ & 5.67 & Christophorou et al. ${ }^{53}$ \\
\hline$e+\mathrm{CF}_{4} \rightarrow \mathrm{CF}_{2}+2 \mathrm{~F}+e$ & $2.221 \times 10^{-10} T_{e}^{0.9939} \exp \left(-14.775 / T_{c}\right)$ & 9.32 & Christophorou el $a l^{53}$ \\
\hline$e+\mathrm{CF}_{4} \rightarrow \mathrm{CF}+3 \mathrm{~F}+e$ & $7.289 \times 10^{-9} T_{e}^{-0.1653} \exp \left(-28.261 / T_{e}\right)$ & 14.7 & Christophorou ef $a .^{53}$ \\
\hline$e+\mathrm{CF}_{3} \rightarrow \mathrm{CF}_{2}+\mathrm{F}+e$ & $3.257 \times 10^{-9} T_{e}^{0.6906} \exp \left(-3.65 / T_{e}\right)$ & 3.65 & Christophorou et al..$^{536}$ \\
\hline$e+\mathrm{CF}_{3} \rightarrow \mathrm{CF}+2 \mathrm{~F}+e$ & $2.221 \times 10^{-10} T_{e}^{0.9939} \exp \left(-9.04 / T_{e}\right)$ & 9.04 & Christophorou et al..$^{93 \mathrm{~b}}$ \\
\hline$e+\mathrm{CF}_{2} \rightarrow \mathrm{CF}+\mathrm{F}+e$ & $3.257 \times 10^{-9} T_{e}^{0.6906} \exp \left(-5.39 / T_{e}\right)$ & 5.39 & Christophorou et al..$^{53 \mathrm{~b}}$ \\
\hline$e+\mathrm{CF} \rightarrow \mathrm{C}+\mathrm{F}+e$ & $3.257 \times 10^{-9} T_{c}^{0.6906} \exp \left(-5.69 / T_{e}\right)$ & 5.69 & Christophorou pt al..$^{\mathrm{sb}}$ \\
\hline$e+\mathrm{F}_{2} \rightarrow \mathrm{F}+\mathrm{F}+e$ & $3.931 \times 10^{-9} T_{e}^{1.2270} \exp \left(-2.544 / T_{e}\right)$ & 1.65 & Edelson and Flammm ${ }^{56}$ \\
\hline$e+\mathrm{CF}_{3}^{+} \rightarrow \mathrm{CF}_{2}+\mathrm{F}$ & $6 \times 10^{-8}$ & -5.44 & Nighan and Wiegand ${ }^{570}$ \\
\hline$e+\mathrm{CF}_{2}^{+}+\mathrm{CF}+\mathrm{F}$ & $6 \times 10^{-8}$ & -6.10 & Nighan and Wiegand ${ }^{57 c}$ \\
\hline$e+\mathrm{CF}^{+} \rightarrow \mathrm{C}+\mathrm{F}$ & $6 \times 10^{-8}$ & -3.47 & Nighan and Wiegand ${ }^{57 c}$ \\
\hline $\mathrm{CF}_{3}+\mathrm{F}_{2} \rightarrow \mathrm{CF}_{4}+\mathrm{F}$ & $3.882 \times 10^{-12} \sqrt{T} \exp (-0.1256 / T)$ & -4.03 & Modica and Sillers ${ }^{58}$ \\
\hline $\mathrm{CF}_{2}+\mathrm{F}_{2} \rightarrow \mathrm{CF}_{3}+\mathrm{F}$ & $3.542 \times 10^{-11} \sqrt{T} \exp (-0.092 / T)$ & -2.0 & Modica and Sillers ${ }^{5 x}$ \\
\hline $\mathrm{F}^{+}+\mathrm{CF}_{3} \rightarrow \mathrm{F}+\mathrm{CF}_{3}^{+}$ & $1 \times 10^{-9}$ & -8.4 & Xu et al. ${ }^{59}$ \\
\hline $\mathrm{F}^{+}+\mathrm{CF}_{4} \rightarrow \mathrm{F}_{2}+\mathrm{CF}_{3}^{+}$ & $1 \times 10^{-9}$ & -4.37 & Xu ef al. ${ }^{54}$ \\
\hline
\end{tabular}

${ }^{\mathrm{a}} \mathrm{Heat}$ of reaction.

'Estimate based on $\mathrm{CF}_{4}$ analogy.

'Estimate based on similar systems.

stainless steel and is grounded in the present study. The inductive coil is connected to a $13.56 \mathrm{MHz}$ if power supply through a matching network.

The LP measurements were made using a commercially available rf compensated Smart Probe made by Scientific
Systems Inc. The body of the probe is made of a ceramic tube with a tapered ceramic tip holder. A platinum wire 380 $\mu \mathrm{m}$ in diameter and $2.5 \mathrm{~mm}$ in length is chosen for the probe tip. The probe is mounted on a motion feedthrough and inserted into the plasma through one of the radial ports. The

TABLE II. Additional reactions involving negative ions, $\mathrm{F}^{-}$.

\begin{tabular}{|c|c|c|c|}
\hline Reaction & Rate coefficient $\left(\mathrm{cm}^{3} s^{-1}\right)$ & $\Delta H_{\mathrm{r}}^{\mathrm{u}}(\mathrm{eV})^{\mathrm{a}}$ & Reference \\
\hline$e+\mathrm{CF}_{4} \rightarrow \mathrm{CF}_{3}+\mathrm{F}^{-}$ & $1.603 \times 10^{-10} T_{e}^{-0.4289} \exp \left(-4.139 / T_{e}\right)$ & 2.21 & Christophorou et al..$^{53}$ \\
\hline$e+\mathrm{CF}_{3} \rightarrow \mathrm{CF}_{2}+\mathrm{F}^{-}$ & $1.603 \times 10^{-10} T_{e}^{-0.4289} \exp \left(-0.18 / T_{e}\right)$ & 0,18 & Christophorou ef al ${ }^{53 b}$ \\
\hline$\rho+\mathrm{CF}_{2}++\mathrm{CF}+\mathrm{F}^{-}$ & $1.603 \times 10^{-10} T_{e}^{-0.4289} \exp \left(-1.93 / T_{c}\right)$ & 1.93 & Christophorou et al. ${ }^{5,36}$ \\
\hline$e+\mathrm{CF}^{-} \rightarrow \mathrm{C}+\mathrm{F}^{-}$ & $1.603 \times 10^{-10} T_{e}^{-0.4289} \exp \left(-2.22 / T_{e}\right)$ & 2.22 & Christophorou et a!. 536 \\
\hline$e+\mathrm{F}_{2} \rightarrow \mathrm{F}+\mathrm{F}^{-}$ & $3.974 \times 10^{-9} T_{e}^{-1.3759} \exp \left(-0.2256 / T_{e}\right)$ & -1.82 & Hayashi and Nimura ${ }^{600}$ \\
\hline$e+\mathrm{CF}_{4} \rightarrow \mathrm{CF}_{3}^{+}+\mathrm{F}^{-}+e$ & $5.263 \times 10^{-12} T_{e}^{0.7677} \exp \left(-14.687 / T_{e}\right)$ & 11.3 & Christophorou et al. ${ }^{53}$ \\
\hline$e+\mathrm{CF}_{3} \rightarrow \mathrm{CF}_{2}^{+}+\mathrm{F}^{-}+e$ & $5.263 \times 10^{-12} T_{e}^{0.7677} \exp \left(-11.678 / T_{e}\right)$ & 11.7 & Christophorou et al..$^{53 \mathrm{~b}}$ \\
\hline$e+\mathrm{F}^{-} \rightarrow \mathrm{F}+2 e$ & $5.456 \times 10^{-10} T_{e}^{1.175} \exp \left(-3.296 / T_{e}\right)$ & 3.47 & Edelson and Flamm 56 \\
\hline $\mathrm{CF}_{4}+\mathrm{F}^{-} \rightarrow \mathrm{CF}_{4}+\mathrm{F}+e$ & $2.132 \times 10^{-8} T_{i}^{1.131} \exp \left(-1.448 / T_{i}\right)$ & 3.47 & Peko el al. ${ }^{61}$ \\
\hline $\mathrm{CF}_{3}+\mathrm{F}^{-} \rightarrow \mathrm{CF}_{4}+e$ & $5 \times 10^{-10}$ & -2.21 & Nighan and Wiegand ${ }^{5 \%}$ \\
\hline $\mathrm{CF}_{2}+\mathrm{F}^{-} \rightarrow \mathrm{CF}_{3}+e$ & $5 \times 10^{-10}$ & -0.18 & Nighan and Wiegand ${ }^{5 \%}$ \\
\hline $\mathrm{CF}+\mathrm{F}^{-} \rightarrow \mathrm{CF}_{2}+e$ & $5 \times 10^{-10}$ & -1.93 & Nighan and Wiegand ${ }^{57}$ \\
\hline $\mathrm{C}+\mathrm{F}^{-} \rightarrow \mathrm{CF}+e$ & $5 \times 10^{-10}$ & -2.22 & Nighan and Wie gand $5 \%$ \\
\hline $\mathrm{F}+\mathrm{F}^{-} \rightarrow \mathrm{F}_{2}+e$ & $1.4 \times 10^{-10} \exp \left(-1.9 / T_{i}\right)$ & 1.82 & Mandl $^{62}$ \\
\hline $\mathrm{CF}_{3}^{+}+\mathrm{F}^{-} \rightarrow \mathrm{CF}_{3}+\mathrm{F}$ & $8.8 \times 10^{-7}$ & -5.62 & Hebner and Miller ${ }^{\mathrm{s}}$ \\
\hline $\mathrm{CF}_{2}^{+}+\mathrm{F}^{-} \rightarrow \mathrm{CF}_{2}+\mathrm{F}$ & $8.8 \times 10^{-7}$ & -8.03 & Hebner and Miller ${ }^{63}$ \\
\hline $\mathrm{CF}^{+}+\mathrm{F}^{-}-\mathrm{CF}+\mathrm{F}$ & $8.8 \times 10^{-7}$ & -5.70 & Hebner and Miller ${ }^{63}$ \\
\hline $\mathrm{F}^{+}+\mathrm{F}^{-} \rightarrow \mathrm{F}+\mathrm{F}$ & $4.0 \times 10^{-7}$ & -14.0 & Nighan and Wiegand ${ }^{5 \%}$ \\
\hline
\end{tabular}

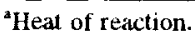

${ }^{b}$ Estimate based on $\mathrm{CF}_{4}$ analogy.

'Estimate based on similar systems. 
TABLE III. Parameters for transport properties.

\begin{tabular}{lccc}
\hline & \multicolumn{2}{c}{ Lennard-Jones parameters } & \\
\cline { 2 - 3 } Molecule & $\begin{array}{c}\text { Vanishing distance, } \\
\sigma(\AA)\end{array}$ & $\begin{array}{c}\text { Well depth, } \\
\epsilon / k(\mathrm{~K})\end{array}$ & $\begin{array}{c}\text { Polarizability } \\
\alpha\left(\AA^{\mathbf{3}}\right)\end{array}$ \\
\hline $\mathrm{CF}_{4}$ & 4.662 & 134 & 2.82 \\
$\mathrm{CF}_{3}$ & 4.320 & 121 & $2.82^{\mathrm{b}}$ \\
$\mathrm{CF}_{2}$ & 3.977 & 108 & $2.82^{\mathrm{b}}$ \\
$\mathrm{CF}$ & 3.635 & 94.2 & $1.8^{\mathrm{c}}$ \\
$\mathrm{F}$ & 2.968 & 112.6 & 0.6 \\
$\mathrm{C}$ & 3.385 & 30.6 & 1.5 \\
$\mathrm{~F}_{2}$ & 3.357 & 112.6 & $0.9^{\mathrm{b}}$ \\
\hline \hline
\end{tabular}

${ }^{\text {"See Ref. } 48 .}$

${ }^{b}$ Estimate based on $\mathrm{CF}_{4}$ analogy.

${ }^{\mathrm{E}}$ Estimate based on $\mathrm{CO}$ analogy.

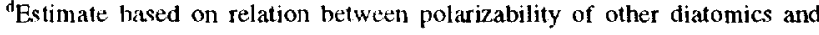
their atoms.

motion of the probe is provided by a computer-controlled stepper motor. The probe tip is cleaned between each data point collection by positively pulse biasing the probe tip so that the tip becomes white hot and desorbs deposited species. Data are taken at seven radial locations from the center of the chamber to $6 \mathrm{~cm}$ in $1 \mathrm{~cm}$ increments. The probe was axially positioned in the center of the plasma between the quartz window and the lower electrode.

The effective electron temperature and density are determined by the appropriate weighted integration of the electron energy distribution function (EEDF), calculated from the second derivative of the probe $I-V$ characteristic. ${ }^{54}$ The EEDF is discretized at 40-50 points, each of which is averaged over 1000 measured data points. The uncertainty of the LP measurements is estimated to be $15 \%$ for the electron density and $\pm 0.5 \mathrm{eV}$ for the electron temperature.

\section{RESULTS AND DISCUSSION}

\section{A. Plasma characteristics}

To illustrate the plasma characteristics, two-dimensional contour plots of the properties throughout the reactor for the

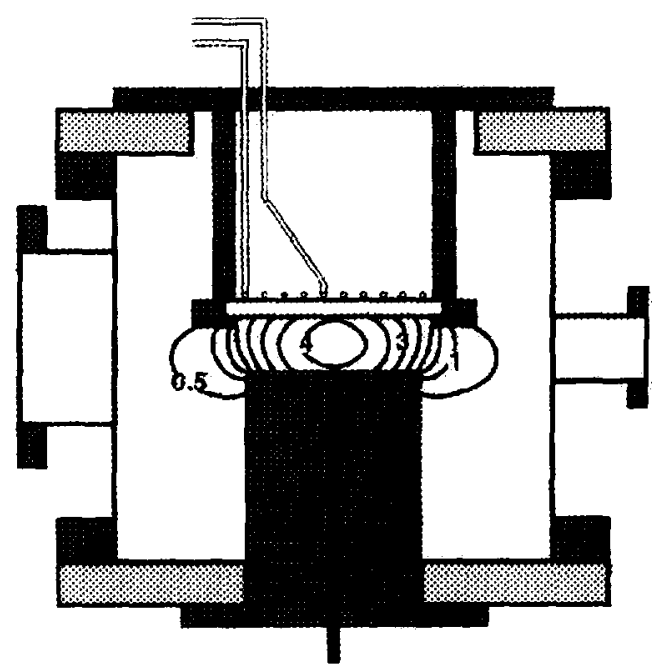

FIG. 1. Contours of electron number density $\left(10^{11} \mathrm{~cm}^{-3}\right)$ at 50 mTorr from SEMS simulation.

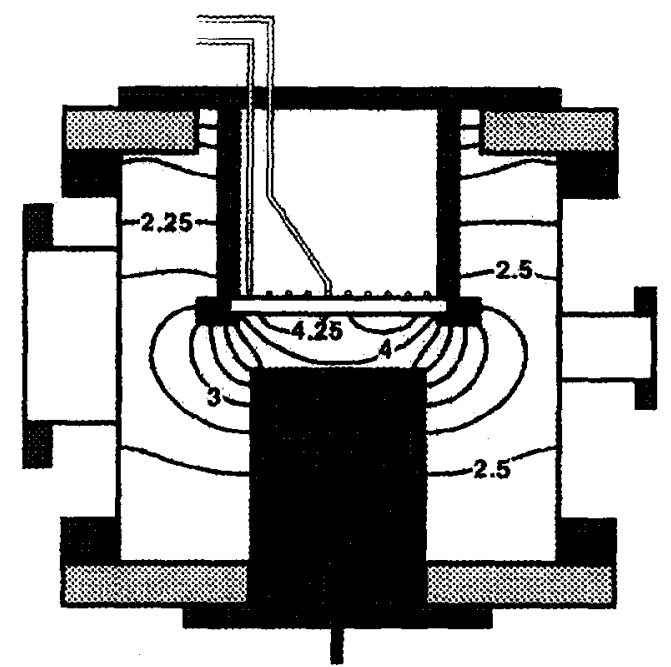

FIG. 2. Contours of electron temperature (eV) at 50 mTort from SEMS simulation.

50 mTorr case are presented. Figure 1 displays the electron number density profiles in the ICP reactor at this pressure. The peak density is $4.25 \times 10^{11} \mathrm{~cm}^{-3}$ in the center of the plasma. An off-axis maximum is not predicted in the simulation, nor is it observed in the experiments (compare Fig. 7). Figure 2 shows the electron temperature. The toroidal shape of the temperature profile also seen in the experimental measurements corresponds to the toroidal power deposition of the ICP.

Figure 3 illustrates the contours of the $\mathrm{CF}_{4}$ mole fraction which show significant consumption of the feed gas in the plasma chamber through various electron impact reactions. Figure 4 displays the $\mathrm{CF}_{3}$ density indicating higher concentrations of the radical near the quartz window and the lower electrode. These higher concentrations result from electron impact dissociation of $\mathrm{CF}_{4}$ and neutralization of the dominant ion, $\mathrm{CF}_{3}^{+}$, at the surfaces (see Fig. 5). $\mathrm{CF}_{3}^{+}$is formed in the chamber by electron impact of the feed gas as well as the

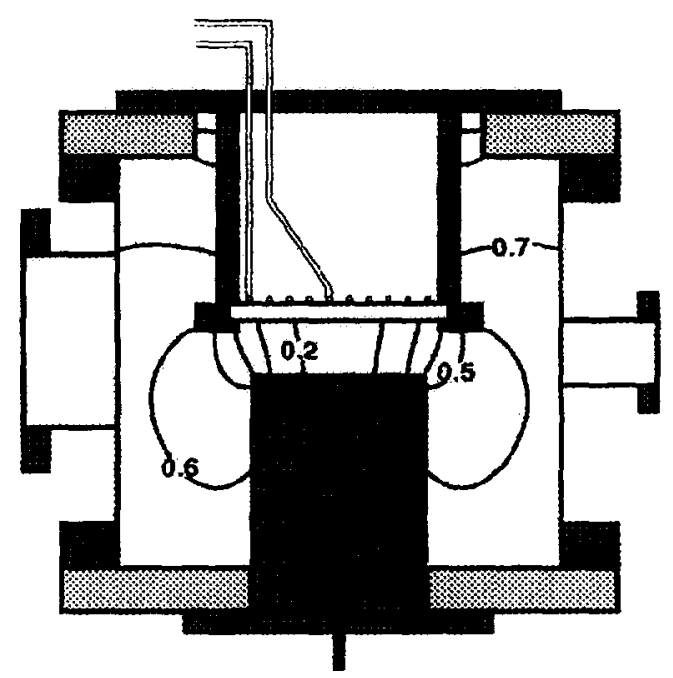

FIG. 3. Contours of $\mathrm{CF}_{4}$ mole fraction at 50 mTorr from sEMs simulation. 


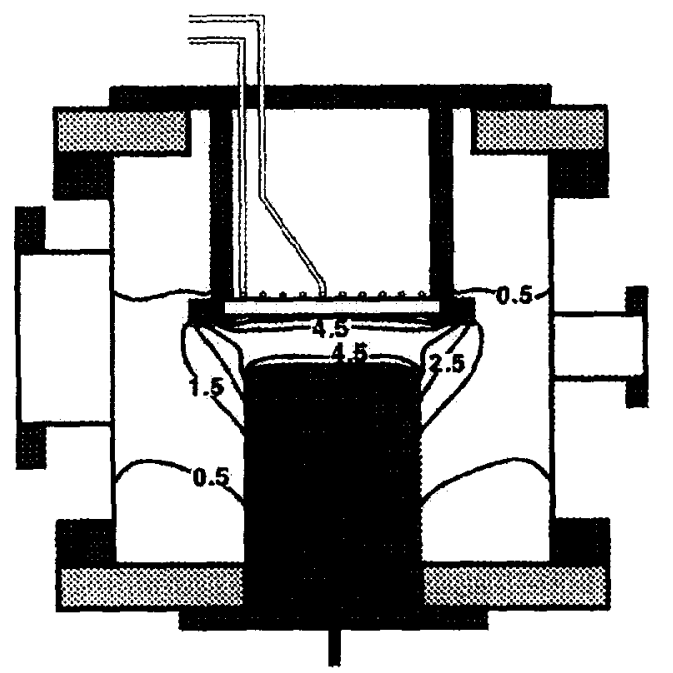

FIG. 4. Contours of $\mathrm{CF}_{3}$ number density $\left(10^{13} \mathrm{~cm}^{-3}\right)$ at 50 mTorr from SEMS simulation.

$\mathrm{CF}_{3}$ radical and then neutralizes upon impact with the quartz window and lower electrode. The peak $\mathrm{CF}_{3}^{+}$density in the central region of the plasma corresponds to the peak electron density in Fig. 1. The predictions reveal that $\mathrm{CF}_{3}^{+}$is the dominant ion.

\section{B. Experimental comparison}

Figure 6 compares the SEMS results using the reaction set in Table I to the radial Langmuir probe measurements for electron temperature made halfway between the quartz window and the lower electrode. The results are plotted from the axis of the reactor to a distance of $8 \mathrm{~cm}$ from the axis. The lower electrode extends out to $5.08 \mathrm{~cm}$. Included with this comparison are drift-diffusion results where gas flow and heating are ignored. For the latter, the neutral gas thermal energy equation and the total momentum equation are re-

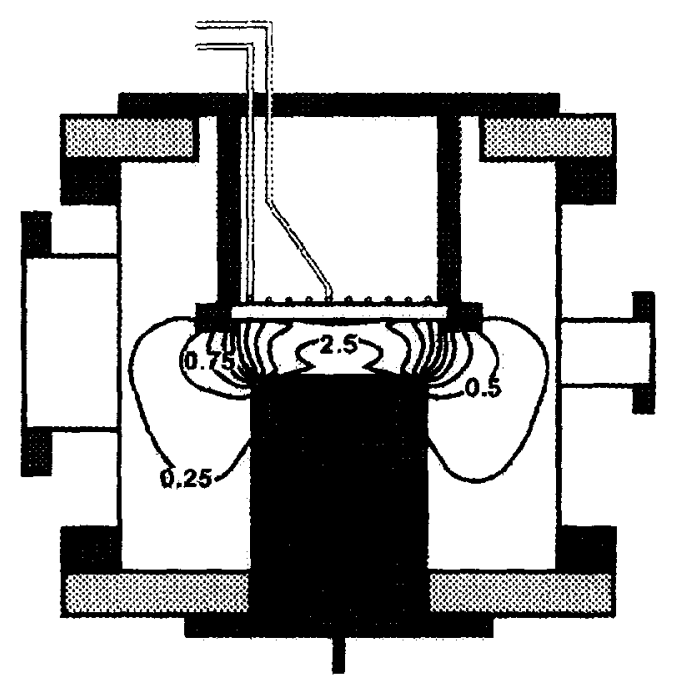

FIG. 5. Contours of $\mathrm{CF}_{3}^{+}$number density $\left(10^{11} \mathrm{~cm}^{-3}\right)$ at 50 miTorr from SEMS simulation.

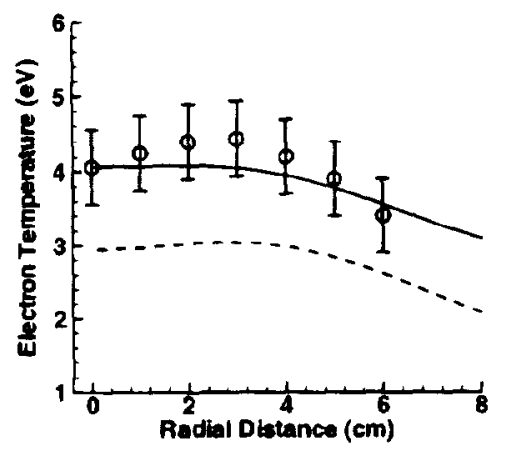

(a)

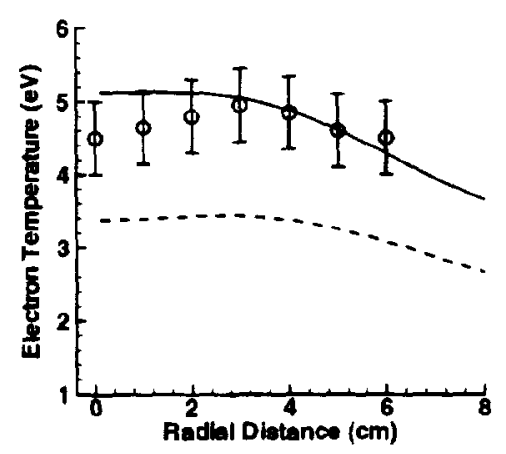

(b)

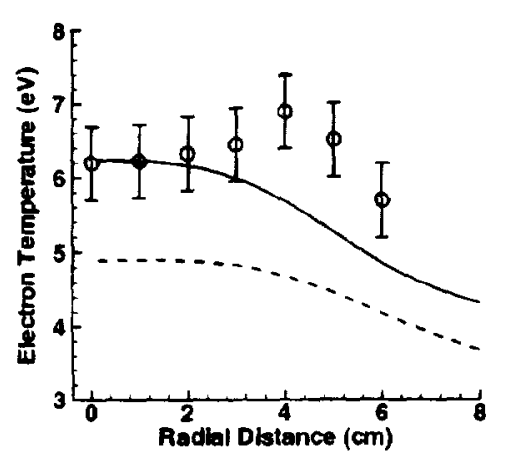

(c)

FIG. 6. Comparison of electron temperature from Langmuir probe measurements (at the axial mid-plane) and simulations: (a) $50 \mathrm{~m}$ Torr, (b) $30 \mathrm{mTorr}$, and (c) 10 mTorr. Simulations using approximate drift-diffusion formulation (dashed lines) and the complete model including gas flow and heating (solid lines) are both compared against experiment (circles).

moved, and the species continuity equations are replaced by the drift-diffusion assumption in which convection is neglected. In addition, the total density, gas pressure, and gas temperature are assumed constant throughout the reactor for the drift-diffusion case. It is seen from the figure that the electron temperature rises with a decrease in pressure as expected. In all three cases, the full model including the effects of gas flow and gas heating predicts the experimental results better than the drift-diffusion approximation. Inclusion of the gas heating effect in the full model is primarily responsible for this predictive capability. Note that, unlike in the larger commercial reactors with substantial flow rates, the pressure drop across the GEC cell is minimal and hence its impact on gas flow and gas density variation is negligible. Therefore, 


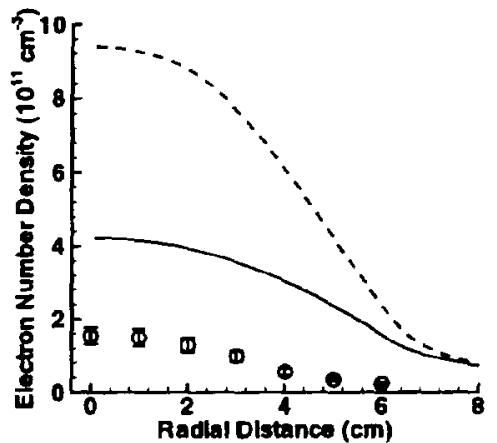

(a)

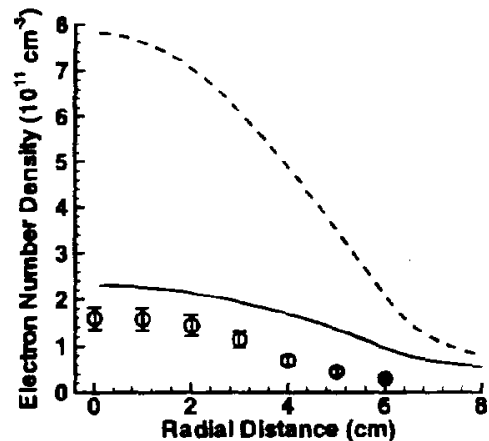

(b)

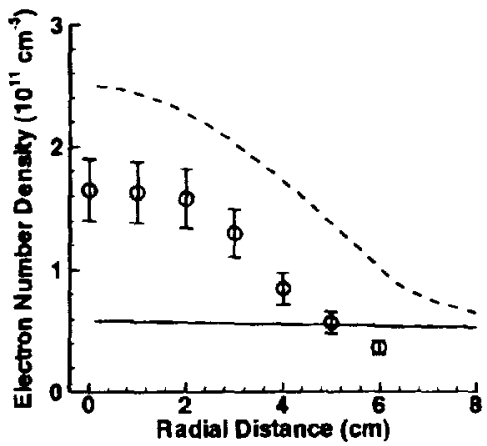

(c)

FIC. 7. Comparison of electron number density from Langmuir probe measurements (at the axial mid-plane) and simulations: (a) $50 \mathrm{mTorr}$ (b) 30 $\mathrm{m}$ Torr, and (c) $10 \mathrm{~m}$ Torr. Simulations using approximate drift-diffusion formulation (dashed lines) and the complete model including gas flow and heating (solid lines) are both compared against experiment (circles).

the increased gas temperature due to charge exchange collisions and dissociative recombination directly results in a decrease in neutral gas densities and in general in a variation of neutral gas density across the reactor. The decrease in gas density results in lower electron impact production rates, leading to a reduced electron density and also results in an increase in electron temperature, relative to the driftdiffusion approximation with its fixed gas temperature and neutral gas density assumptions.

Figure 7 compares the sems results to the Langmuir probe measurements for electron number density. Once again the drift-diffusion results are included. The predictions from the full model of SEMS compare reasonably well with the experiments and better than that of the drift-diffusion ap-

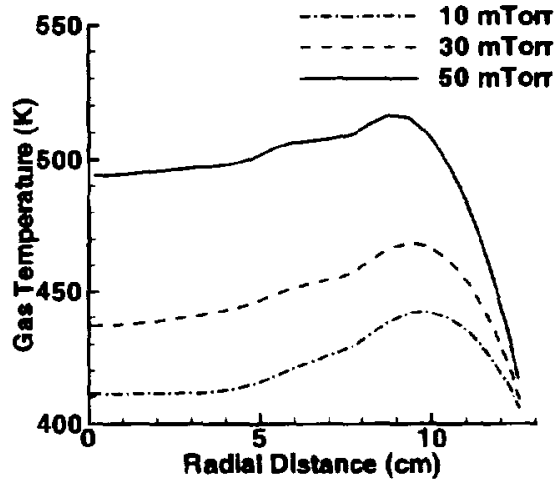

FIG. 8. Radial protiles of gas temperature at the axial mid-plane.

proximation. The experimental results for the 10 mTorr case fall midway between the predictions of the full model and the drift-diffusion model. As the collision frequency decreases with pressure, gas heating becomes less important at low pressures resulting in less difference between the full model and the drift-diffusion model for the 10 mTorr case than the higher pressure cases. The reduction in collision frequency means less exothermic reactions that funnel energy from the plasma to the gas. The decrease in gas temperature with pressure is clearly seen in Fig. 8 which displays the radial profiles of the gas temperature from the center of the plasma to the reactor wall along the axial plane located at the midpoint of the plasma. The peak neutral gas temperature falls from $550 \mathrm{~K}$ at 50 mTorr to $490 \mathrm{~K}$ at 30 mTorr and then to $460 \mathrm{~K}$ at $10 \mathrm{mTorr}$. Also, in Fig. 7, note that the drift-diffusion model consistently predicts higher densities than the full model (and the experiment) which is an artifact resulting from the fixed lower neutral gas temperature $(400 \mathrm{~K})$ and the resulting higher neutral density in the drift-diffusion approximation as discussed earlier.

Although in some low aspect ratio ICPs off-axis maximums in electron density can be seen, this is not the case here in either the experimental measurements or the SEMS predictions. The simulations do a good job of predicting the trends of the density profiles including on-axis maximums. The experimental comparison of electron density with predictions is not as good as for the electron temperature. This is often the case in modeling given the uncertainty in the power absorption efficiency. It is well known that electron temperature does not vary much with power while electron density is strongly power dependent. The assumption of $80 \%$ power absorption we have used may not be completely accurate, resulting in the discrepancies. In fact, later measurements suggest that it is closer to $70 \%$. Another possible reason is that the electron impact rate constants (in Table l) were obtained by integrating the cross sections using a Maxwellian EEDF while the probe measurements ${ }^{40}$ indicate a nonMaxwellian EEDF.

\section{Effect of negative lons}

All of the simulations discussed above were also repeated with the inclusion of negative ions $\left(\mathrm{F}^{-}\right)$and the corresponding reaction set in Table II. Figure 9 shows the effect 


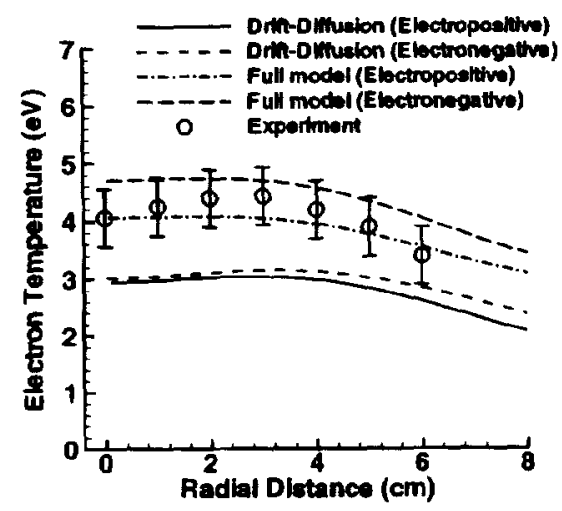

FIG. 9. Effect of negative ions on electron temperature predictions at 50 mTorr.

of including the electronegative reactions on electron temperature for the $50 \mathrm{mT}$ Trr case. The sEMS results including the effects of gas flow and heating show an increase in electron temperature, although the results still fall within the experimental error. This increase is a result of the increased heating from the highly exothermic reactions of ion-ion recombination, which efficiently pump energy from the plasma into the neutral gas. As described previously, elevated gas temperatures result in lower neutral densities, which result in lower electron impact production. This produces a lower electron density, and a higher electron temperature. The driftdiffusion model, with the fixed gas temperature approximation, does not model this effect and thus only exhibits slight differences with the inclusion of negative ions; the small change seen is solely due to differences in radical densities resulting from the electronegative reactions.

Figure 10 illustrates the effect of including the electronegative reactions on the neutral gas temperature. The gas temperature now reaches a maximum of $670 \mathrm{~K}$ compared to 550 $\mathrm{K}$ for the case without negative ions. Moreover, the profiles are different. Without negative ions, the peak heating is outside the active plasma region where significant dissociative

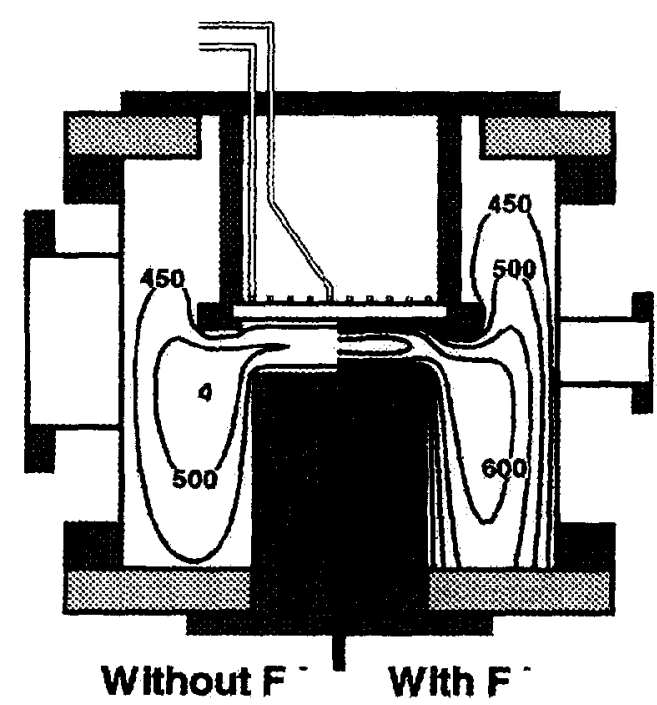

FIG. 10. Effect of negative ions on gas temperature $(K)$ at $50 \mathrm{mTorr}$. recombination takes place. In contrast, the peak heating is seen at the center of the chamber when negative ions are included due to the highly exothermic ion-ion recombination reactions. The rate of these reactions in the central plasma volume is high due to the high local densities of both positive and negative ions.

The 50 mTorr case predicts a plasma with the ratio of number of negative ions to electrons less than one $\left(n_{-} / n_{e}\right.$ $<1$ ). However, the $30 \mathrm{mTorr}$ case predicts a plasma with a ratio greater than one $\left(n_{-} / n_{e}>1\right)$ in the plasma chamber. This highly overpredicted negative ion density results in an erroneous electron temperature mid-plasma radial profile with an axis value of $10 \mathrm{eV}$, more than twice that of the experimentally measured value. The authors are lead to the conclusion that the electronegative reaction mechanisms are suspect. This was further evidenced when it was found that the 10 mTorr case was not possible to simulate with the SEMs code using the electronegative reaction set. Due to the overprediction of negative ions, it was impossible to maintain the plasma.

In the experimental work, biasing on the Langmuir probe could induce instabilities, suggesting the conditions are close to an unstable operating regime. Under some experimental conditions (typically higher pressure and lower power), the discharge was in fact seen to extinguish of switch to capacitive coupling mode. This type of instability has been associated with the presence of negative ions, ${ }^{55}$ and is not inconsistent with the effect of high negative ion concentrations observed in the SEMS code. However, the SEMS predictions in this regard are not completely consistent with the experimental data as it is possible to obtain operating conditions at $10 \mathrm{mTorr}$ and $300 \mathrm{~W}$ without this instability, and the instability is greater at higher, not lower, pressures. This discrepancy in trends further calls into question the accuracy of our electronegative reaction set.

The experimental measurements presented in Fig. 6 may indicate some presence of negative ions. Otherwise, it would be expected that the toroidal shape of the electron temperature profile would dissipate with decreasing pressure as the electron thermal conductivity correspondingly increases. As a result, the off-axis maximums in the radial electron temperature profile should become less pronounced and move toward the axis. Instead, in the experiment, the off-axis maximums may be maintained because of localized production of negative ions due to the toroidal deposition as shown in Fig. 11. The resulting localized depletion of electrons could sustain the off-axis maximums in the electron temperature. Further experimental and computational investigations into the presence of negative ions in pure $\mathrm{CF}_{4}$ plasmas are warranted and will be the subject of future work.

An alternate and/or complementary explanation may arise from the fact that the EEDF is non-Maxwellian contrary to the assumptions of our model. It has been shown in research by Kortshagen et al. ${ }^{64}$ that the EEDF has some bearing on the radial profiles of electron temperature. This may also contribute to the discrepancies in the radial trends predicted by SEMs and measured experimentally. 


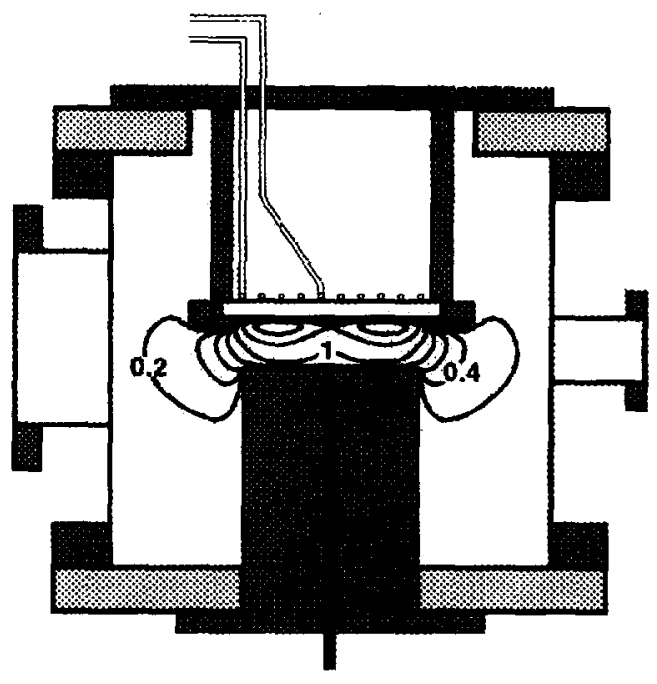

FIG. 11. Contours of $\mathrm{F}^{-}$number density $\left(10^{11} \mathrm{~cm}^{-3}\right)$ at $50 \mathrm{mTort}$ from SEMS simulation.

\section{CONCLUDING REMARKS}

The demands in equipment and process design, arising from the continuous scaling down of device feature sizes, scaling up of reactor dimensions, and from the increased costs of new fabs, have made modeling and diagnostics valuable in integrated circuit manufacturing. Development of technology computer aided design tools require accurate models that capture the process physics and chemistry. In plasma processing, models have historically done well in capturing the discharge physics and power coupling features but overlooked issues related to the neutral gas. In the present work, we have used the SEMS code, which includes gas flow and gas heating models coupled to plasma genera-

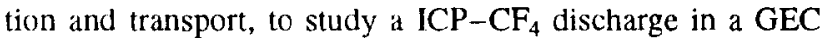
reference plasma reactor. The analysis shows significant heating of the gas and temperature variations across the reactor which affect the plasma characteristics and radical densities. We have also measured the electron density and temperature in the GEC reactor using a Langmuir probe. The predictions agree well with the measurements. When the full model in SEMS is relaxed to the familiar drift-diffusion formulation, by dropping the Navier-Stokes and gas-energy equations and assuming a constant gas density and temperature throughout the reactor, the model results depart more from the measurements. Finally, the experiment could indicate some presence of negative ions and/or the need for more accurate modeling of the EEDF through the use of a Boltzmann solver. This would likely improve the quality of our comparisons to the experiment in regard to the prediction of the off-axis maximums in electron temperature. However, current knowledge of negative ion reaction kinetics is suspect, and further detailed research into the actual electronegative reaction mechanisms and cross sections is warranted.

\section{ACKNOWLEDGMENTS}

D.B.H., D.B., M.V.V.S.R., and B.A.C.'s work was conducted while employed by Eloret Corporation supported un- der NASA Contract No. NAS2-99092. The authors would also like to acknowledge helpful discussions with Mark Kiehlbauch and Professor David Graves at University of California, Berkeley.

${ }^{1}$ F. F. Young and C.-H. Wu, Appl. Phys. Lett. 62, 473 (1993).

${ }^{2}$ J.-H. Tsai and C.-H. Wu, J. Phys. D 26, 496 (1993).

${ }^{3}$ P. L. G. Ventzek, T. J. Sommerer, R. J. Hoekstra, and M. J. Kushner, Appl. Phys. Lett. 63, 605 (1993).

${ }^{4}$ P. L. G. Ventzek, R. J. Hoekstra, and M. J. Kushner, J. Vac. Sci. Technol B 12, 461 (1994).

${ }^{5}$ P. L. G. Ventzek, M. Grapperhaus, and M. J. Kushner, J. Vac. Sci. Technol. B 12, 3118 (1994).

${ }^{6} \mathrm{~W}$. Z. Collison and M. J. Kushner, Appl. Phys. Lett. 68, 903 (1996).

${ }^{7}$ M. J. Kushner, W. Z. Collison, M. Grapperhaus, J. P. Holland, and M. S. Bames, J. Appl. Phys. 80, 1337 (1996).

${ }^{8}$ M. Grapperhaus and M. J. Kushner, J. Appl. Phys. 81. 569 (1997)

${ }^{9}$ S. Rauf and M. J. Kushner, J. Appl. Phys. 81, 5966 (1997).

${ }^{10} \mathrm{~S}$. Rauf and M. J. Kushner, J. Appl. Phys. 83, 5087 (1998).

${ }^{11}$ A. Fiala, L. C. Pitchford, and J. P. Boeuf, Phys. Rev. E 49, 5607 (1994).

${ }^{12}$ D. B. Graves, H.-M. Wu, and R. K. Porteus, Jpn. J. Appl. Phys., Part 132. 2999 (1993).

${ }^{13}$ R. K. Porteous, H.-M. Wu, and D. B. Graves, Plasma Sources Sci. Technol. 3, 25 (1994).

${ }^{14}$ H.-M. Wu, D. B. Graves, and R. K. Porteous, Plasma Sources Sci. Technol. 4, 22 (1995).

${ }^{15}$ F. F. Young and C.-H. Wu, IEEE Trans. Plasma Sci. 21, 312 (1993)

${ }^{16}$ F. F. Young and C.-H. Wu, J. Appl. Phys. 74, 839 (1993).

${ }^{17}$ M. Dalvie, M. Surendra, and G. S. Selwyn, Appl. Phys. Lett. 62, 3207 (1993).

18 J. D. P. Passchier and W. J. Goedheer, J. Appl. Phys. 74, 3744 (1993).

${ }^{19}$ W. J. Goedheer, P. M. Meijer, J. Bezemer, J. D. P. Paschier, and W. G. J. H. M. van Sark, IEEE Trans. Plasma Sci. 23, 644 (1995).

${ }^{20}$ D. P. Lymberopoulos and D. J. Economou, Appl. Phys. Lett. 63, 2478 (1993).

${ }^{21}$ D. P. Lymberopoulos and D. J. Economou, J. Vac. Sci. Technol. A 12, 1229 (1994).

${ }^{22}$ D. P. Lymberopoulos and D. J. Economou, IEEE Trans. Plasma Sci. 23, 573 (1995).

${ }^{23}$ D. P. Lymberopoulos and D. J. Economou, J. Res. Natl. Inst. Stand. Technol. 100, 473 (1995).

${ }^{24}$ R. S. Wise, D. P. Lymberopoulos, and D. J. Economou. Appl. Phys. Lett. 68, 2499 (1996).

${ }^{25}$ R. A. Stewart, P. Vitello, and D. B. Graves, J. Vac. Sci. Technol. B 12, 478 (1994).

${ }^{26}$ R. A. Stewart, P. Vitello, D. B. Graves, E. F. Jaeger, and L. A. Berry, Plasma Sources Sci. Technol. 4, 36 (1995)

${ }^{27}$ A. Fiala, M. Kiehlbauch, S. Mahnoyski, and D. B. Graves, J. Appl. Phys. 86, 152 (1999).

${ }^{28}$ M. Li, H.-M. Wu, and Y. Chen, IEEE Trans. Plasma Sci. 23, 558 (1995).

${ }^{29}$ H.-M. Wu, B. W. Yu, M. Li, and Y. Yang, IEEE Trans. Plasma Sci. 25. 1 (1997).

${ }^{30}$ H.-M. Wu, B. W. Yu, A. Krishnan, M. Li, Y. Yang, J.-P. Yan, and D.-P. Yuan, IEEE Trans. Plasma Sci. 25, 776 (1997).

${ }^{3 !}$ M. H. Wilcoxson and V. I. Manousiouthakis, Chem. Eng. Sci. 51, 1089 (1996).

${ }^{32}$ K. Bera, B. Farouk, and Y. H. Lee, Plasma Sources Sci. Technol. 8, 412 (1999).

${ }^{33}$ K. Bera, B. Farouk, and Y. H. Lee, Plasma Sources Sci. Technol. 10, 1 (2001).

${ }^{34}$ M. H. Khater, L. J. Overzet, and B. E. Cherrington, J. Vac. Sci. Technol. B 16, $490(1998)$

${ }^{35}$ G. A. Hebner, J. Appl. Phys, 80, 2624 (1996).

${ }^{36}$ M. V. Malyshev, V. M. Donnelly, S. W. Downey, J. I. Colonell, and N. Layadi, J. Vac. Sci. Technol. A 18, 849 (2000)

${ }^{37}$ M. W. Kiehlbauch and D. B.Graves, J. Appl. Phys. 89, 2047 (2001)

${ }^{38}$ J. D. Bukowski, D. B. Graves, and P. Vitello, J. Appl. Phys. 80, 2614 (1996).

${ }^{39} \mathrm{~S}$. K. Nam, C. H. Shin, and D. J. Econonou, Mater. Sci. Senicond. Process. 2, 271 (1999).

${ }^{40}$ M. V. V. S. Rao, S. P. Sharma, B. A. Cruden, and M. Meyyappan, J. Vac. Sci. Technol. A (submitted). 
${ }^{41}$ B. A. Cruden, M. V. V. S. Rao, S. P. Shama, and M. Meyyappan, Plasma Sources Sci. Technol. (to be published).

${ }^{42}$ D. Bose, T. R. Govindan, and M. Meyyappan, J. Electrochem. Soc. 146 , 2705 (1999)

${ }^{43}$ D. Bose, T. R. Govindan, and M. Meyyappan, IEEE Trans. Plasma Sci 27, 54 (1999).

${ }^{44}$ C. H. Chang and D. Bose, IEEE Trans. Plasma Sci. 27, 1310 (1999).

${ }^{45}$ J. D. Ramshaw and C. H. Chang, Plasma Chem. Plasma Process. 11, 395 (1991).

46 J. D. Ramshaw and C. H. Chang, Plasma Chem. Plasma Process. 13, 489 (1993).

${ }^{47}$ J. D. Ramshaw and C. H. Chang, Phys. Rev. E 53, 6382 (1996).

${ }^{48}$ E. W. McDaniel and E. A. Mason, The Mobility and Diffusion of lons in Gases (Wiley, New York, 1973).

${ }^{49}$ D. Bose, T. R. Govindan, and M. Meyyappan, IEEE Trans. Plasma Sci. (submitted).

${ }^{50}$ M. Mcyyappan and T. R. Govindan, J. Appl. Phys. 80, 1345 (1996).

${ }^{51}$ G. P. Kota, J. W. Coburn, and D. B. Graves, J. Vac. Sci. Technol. A 17, 282 (1999).
${ }^{52}$ R. N. Gupta, J. M. Yos, R. A. Thompson, and K. Lee, NASA Ref. Pub. No. 1232, August 1990 (unpublished).

${ }^{53}$ L. G. Christophorou, J. K. Olthoff, and M. V. V. S. Rao, J. Phys. Chem. Ref. Data 25, 1341 (1996).

${ }^{54}$ J. S. Kim, M. V. V. S. Rao, M. A. Cappelli, S. P. Sharma, and M. Meyyappan, Plasma Sources Sci. Technol, 110, 191 (2001).

${ }^{55}$ M. A. Lieberman, A. J. Lichtenberg, and A. M. Marakhtanov, Appl. Phys. . Lett. 75, 3617 (1999).

${ }^{58}$ D. Edelson and D. L. Flamm, J. Appl. Phys. 56, 1522 (1984).

${ }^{57}$ W. L. Nighan and W. J. Wiegand, Phys. Rev. A 10, 922 (1974).

${ }^{58}$ A. P. Modica and S. J. Sillers, J. Chem. Phys. 48, 3283 (1968).

${ }^{59}$ X. Xu, S. Rauf and M. J. Kushner, J. Vac. Sci. Technol. A 18, 213 (2000).

${ }^{60}$ M. Hayashi and T. Nimura, J. Appl. Phys. 54, 4879 (1983).

${ }^{61}$ B. L. Peko, I. V. Dyakov, R. L. Champion, M. V. V. S. Rao, and J. K. Olthoff, Phys. Rev. E 60, 7449 (1999).

${ }^{62}$ A. Mandl, J. Chem. Phys. 59, 3423 (1973).

${ }^{63}$ G. A. Hebner and P. A. Miller, J. Appl. Phys. 87, 7660 (2000).

${ }^{64}$ U. Kortshagen, C. Busch, and L. D. Tsendin, Plasma Sources Sci. Technol. 5,1 (1996). 